\title{
Mathematical Analysis Power Spectrum of M-ary MSK and Detection with Optimum Maximum Likelihood
}

\author{
Zheng Niu ${ }^{1}$, Yuzhong Jiang ${ }^{*}$, Shuyan g Jia², Zhi Huang ${ }^{1}$, Wenliang Zou ${ }^{1}$, Gang Liu', Yu Li ${ }^{3}$ \\ ${ }^{1}$ College of Electronic Engineering, Naval University of Engineering, Wuhan, People's Republic of China \\ [E-mail: ahhfnz@163.com.jiangyuzhong@tsinghua.org.cn, hzpaperphd@163.com,1668166203@qq.com, \\ 18117616337@163.com] \\ ${ }^{2}$ College of the 2nd department, Navy Submarine Academy, Qindao, People's Republic of China \\ [E-mail: 2443507071@qq.com, \\ ${ }^{3}$ School of Information Engineering, Nanchang University, Nanchang, People's Republic of China \\ [E-mail: 302764948@qq.com] \\ * Corresponding author: Yuzhong Jiang
}

Received February 6, 2021; revised April 4, 2021; revised May 17, 2021; accepted July 3, 2021; published August 31, 2021

\begin{abstract}
Abstact
In this paper, the power spectral density(PSD) for Multilevel Minimum Shift Keyed signal with modulation index $h=1 / 2$ (M-ary MSK) are derived using the mathematical method of the Markov Chain model. At first, according to an essential requirement of the phase continuity characteristics of MSK signals, a complete model of the whole process of signal generation is built. Then, the derivations for autocorrelation functions are carried out precisely. After that, we verified the correctness and accuracy of the theoretical derivation by comparing the derived results with numerical simulations using MATLAB. We also divided the spectrum into four components according to the derivation. By analyzing these figures in the graphic, each component determines the characteristics of the spectrum. It is vital for enhanced spectral characteristics. To more visually represent the energy concentration of the main flap and the roll-down speed of the side flap, the specific out-of-band power of M-ary MSK is given. OMLCD(Optimum Maximum Likelihood Coherent Detection) of M-ary MSK is adopted to compare the signal received with prepared in advance in a code element $\mathrm{T}$ to go for the best. And M-ary MSK BER(Bit Error Rate) is compared with the same ary PSK (Phase Shift Keying) with $M=2,4,6,8$. The results show the detection method could improve performance by increasing the length of L(memory inherent) in the phase continuity.
\end{abstract}

Keywords: M-ary MSK, Power-Spectral-Density, Markov Chain Process Strategy, Optimum Maximum Likelihood Coherent Detection. 


\section{Introduction}

In this paper, we analyzed the M-ary MSK communication system in two aspects: spectral properties of M-ary MSK (Multilevel Minimum Shift Keyed) signal with modulation index $\mathrm{h}=1 / 2$ and optimum performance of optimum maximum likelihood coherent detection in BER. We derive the PSD expression using the mathematical method of the Markov Chain model to make the process more streamlined. The results can help us search the key points specific to a particular formula that affects the spectral characteristics and facilitates spectrum modification. The simulation of M-ary MSK in BER can be used to assess the stability of communication system transmissions. The MSK signals are very widely used in the VLF(Very Low Frequency) communication field. The benefits of the modulated signal possess a consistent envelope and a high bandwidth efficiency. Chen Xingbo [1] changed MSK's carrier to LFM (Linear Frequency Modulation) signal, MSK-LFM. He analyzed the energy leakage of MSK-LFM based on the spectrum in expression and simulation. Dong [2] demonstrated a receiver using PLL(Phase-Locked Loop) to get a high-speed transmission with a low BER. Wang [3] derived the BER of MSK modulation based on the gamma-gamma channel model with coherent detection and analyzed the BER performance of MSK in weak, medium, and strong turbulence. This continuous phase limitation affects the signal with two crucial aspects. At first, with the transient effects, lessened spectral bandwidth will get better. Secondly, the continuous phase will improve demodulation performance by making decisions with several symbols rather than one symbol only [4]. M-ary MSK also has the advantages of MSK apply for Very Low Frequency to ensure no excessive power during phase shift. It can not only increase the communication rate but also improve spectral efficiency. Le Nguyen Binh applied the M-ary MSK modulation scheme for long-haul optically amplified transmission systems. According to the numerical result, a significant increase is achieved in transmission rates [5]. For a variety of MSK-derived communication systems, M-ary MSK is used to improve spectral efficiency. For example, Tao analyzed IRZ-MSK in a high-speed optical fiber transmission system from [6], research can be continued on multi-decimal.

However, using multi-decimal symbols instead of binary symbols within a code element can result in a significant increase in transmission rate [7]. While improving transmission efficiency, analyzing the spectral characteristics has become an essential part. Anderson and Salz [8] considered getting the PSD of M-ary CPFSK(Continuous Phase Frequency Shift Keying) by directly calculating the Fourier transform over a long time interval signal waveform. In addition, we derive the theoretical expressions of M-ary MSK with $h=1 / 2$ and present the compositions of M-ary MSK, which is vital for analyzing the spectrum structure and use in communication system design for improving the performance [9]. Ekanayake [10] derived the spectral properties of M-ary CPFSK with $\mathrm{h}=1 / \mathrm{M}$ by extending reference [9], which the method developed for binary digital FM(Frequency-Modulated) gives the PSD using a wave train made up of sine-wave segments. Obviously, as the binary number 
increases, the modulation index also rises, reducing the phase difference between adjacent characters, thus leading to a higher chance of demodulation errors. Biglieri used the representation of Rimoldi [11] to calculate a closed-form expression for the power spectral density of full-response M-ary CPM with modulation index $\mathrm{J} / \mathrm{M}$ by straightforward computations, $\mathrm{J}$ an integer. The direct derivation process can be tedious and challenging to understand than building a model.

M-ary MSK compared to MSK, the transmission efficiency improves significantly. Considering the characteristics of MSK signals, the spectrum of M-ary MSK is analyzed by the Markov Chain model at the same time. Reasons for MSK modulation process can be built with Markov Chain process for spectral analytical analysis are as follows:

1. The signal sent by the MSK source is completely statistically independent, which corresponds to the Markov Chain with no after-effects.

2. With the fixed modulation index $1 / 2$ and periodicity of trigonometric functions in phase, the number of states is inevitable, corresponding to a Markov Chain state space.

3. The transfer between states can accordingly be expressed in terms of the transfer probabilities of Markov Chains [12,13,14].

Simon and Divsalar [15] have discussed the performance of coherent detection for MPSK and give the structure of making decisions on two successive symbols. However, Park, Lee, and Feher [16], following the CPM(Continuous Phase Modulation) characteristics, proposed an optimum ML(Maximum-Likelihood) coherent receiver for unique CPM signals, while Andersson, Svensson [17] considered a noncoherent detector with an environment of white Gaussian noise, and they proposed a decoding algorithm for these convolutional coding CPM schemes. In 1992, Jung, Baier proposed a new novel signal generation concept for CPM with $h=1 / 2$ based on real-valued impulses. They derived the expressions in mathematical for both the real impulses and the bandpass CPM signals[18].

\section{Signal Expression}

MSK is a special CPFSK with $h=1 / 2$. We can express the i-th phase of M-ary MSK signal as [19]:

$$
\Phi_{i}=\frac{d_{i} \pi h(t-(i-1) T)}{T}+\pi h \sum_{j=1}^{i-1} d_{j}+\theta \quad(i-1) T<t<i T
$$

So the signal can be simplified and written as:

$$
r(t)=A \cos \left(\Phi_{i}\right) \quad(i-1) T<t<i T
$$

where
A The amplitude of the signal
$h \quad$ Modulation index
$d_{i} \quad$ Digital information signal 
$T \quad$ The whole symbol interval

$\theta \quad$ Starting phase

It is clear that when $i=1, \sum_{j=1}^{i-1} d_{j}=0$. We set the modulation index $h=1 / 2$, and the amplitude $A$ is normalized. In each extended interval, one value of numeric information is assumed from the set $\{ \pm 1, \pm 3, \pm 5, \ldots, \pm(M-1)\}$. According to (1), we can see clearly that the difference with PSK modulation is that the current phase is related to the current input information and the phase of the last moment to ensure the phase continuity. All possible phase trajectories for all values of the phase term $\Phi_{i}$ are shown in Fig. 1. For 4-ary MSK modulation, it is assumed that the starting phase $\theta$ is known, so it is generally considered to be 0 , and there is no loss in generality. Therefore we set $\theta=0$ and $d_{i}= \pm 1, \pm 3$. When the next phase with digital information input increases over $2 \pi$ or decreases under $-2 \pi$, it can always be equated to one of the existing state trajectories because of the periodicity of the cosine function.

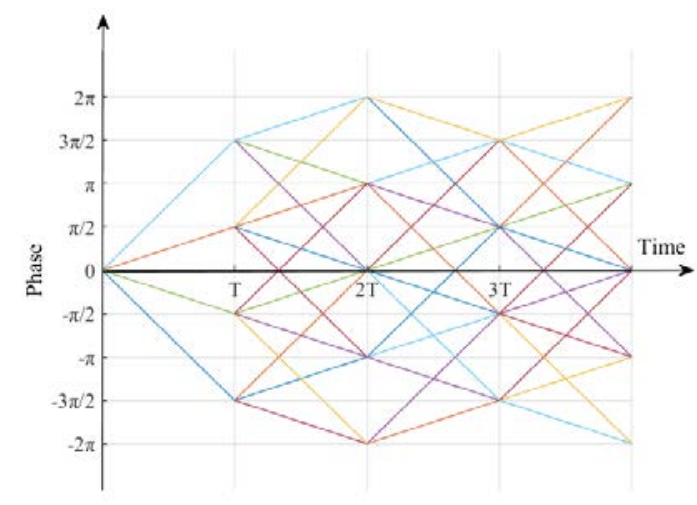

Fig. 1. Phase trellis of 4-ary MSK

\section{The Derivation of M-ary MSK Autocorrelation Function in Mathematical Expressions}

As mentioned in the Introduction, we apply the Markov Chain Process Strategy to derive the analytical expression of the spectrum of M-ary MSK. At first, we evaluate the autocorrelation functions of M-ary MSK. Then we get the theoretical spectrum expressions. Markov Chain Strategy is based on references [13] [20].

\subsection{Markov Chain Process Strategy}

We assumed $X(t)=X_{R}(t)+j X_{I}(t)$ is random M-ary MSK signal because within one symbol interval $T_{b}$ digital information takes one of the set values $\left\{ \pm D_{1}, \pm D_{2}, \pm D_{3}, \ldots, \pm D_{d}\right\}=\{ \pm 1, \pm 3, \pm 5, \ldots, \pm(\mathrm{M}-1)\}$ with equal probability. So that $X_{R}(t)$ and 
$X_{I}(t)$ have $4 M$ types of states in every symbol interval, Those states are:

$$
\begin{gathered}
\sin \Phi\left( \pm D_{1}\right), \sin \Phi\left( \pm D_{2}\right), \sin \Phi\left( \pm D_{3}\right), \ldots, \sin \Phi\left( \pm D_{d}\right) \\
\cos \Phi\left( \pm D_{1}\right), \cos \Phi\left( \pm D_{2}\right), \cos \Phi\left( \pm D_{3}\right), \ldots, \cos \Phi\left( \pm D_{d}\right)
\end{gathered}
$$

Because of the symmetry of the I and Q channels, we only take the actual envelope of the I channel signal for analysis. The types of $X_{R}(t)$ states of each symbol interval are decimated to $2 M$ :

$$
\sin \Phi\left( \pm D_{1}\right), \sin \Phi\left( \pm D_{2}\right), \sin \Phi\left( \pm D_{3}\right), \ldots, \sin \Phi\left( \pm D_{d}\right)
$$

We can simplify the above states by eliminating the positive and negative signs to obtain the following expressions:

$$
\sin \Phi\left(D_{d}\right), \sin \Phi\left(D_{d}+1 / 2 \pi\right), \sin \Phi\left(D_{d}+\pi\right), \sin \Phi\left(D_{d}+3 / 2 \pi\right)
$$

That is:

$$
\sin \Phi\left(D_{d}\right), \cos \Phi\left(D_{d}\right),-\cos \Phi\left(D_{d}\right),-\sin \Phi\left(D_{d}\right)
$$

The states are shown in equation (3) is arranged from 1 to $\mathrm{d}$ by $D_{d}$ and gather each different state groups numbering the states in order states 1 to state $2 M$.

Because the signals sent by the sources are statistically independent and equal in probability. For each of the $2 M$ states, the starting state probability is

$$
P(i)=\frac{1}{N}=\frac{1}{2 M}, i \in[1,2 M] \text {. }
$$

$N$ is the number of starting states. Under a different number of transfers $m, p(j \mid i, m)$ represents the probability of transition from state $i$ to state $j$. Put them into the matrix to get its state transfer matrix $P_{M}{ }^{m}$ :

a) In the case $m=0$ :

$$
P_{M}{ }^{0}=\overbrace{\left(\begin{array}{lll}
1 & & \\
& \ddots & \\
& & 1
\end{array}\right)}^{2 M}
$$

$p(j \mid i, m)$ corresponds to the element $p_{i, j}^{(m)}$ in the matrix $P_{M}^{m}$. The superscript $m$ represents the number of transfer steps, and the subscript $M$ denotes the M-ary.

b) In the case $m=1$ :

When a one-step transfer is performed, the transfer probability of each state can be obtained from the phase initiation, termination characteristics of each state signal, and the phase continuity of the signal. We can get the state transfer matrix at $m=1$. The following Fig. 2 shows the 8-ary MSK state figure, from which the general rule can be obtained. 


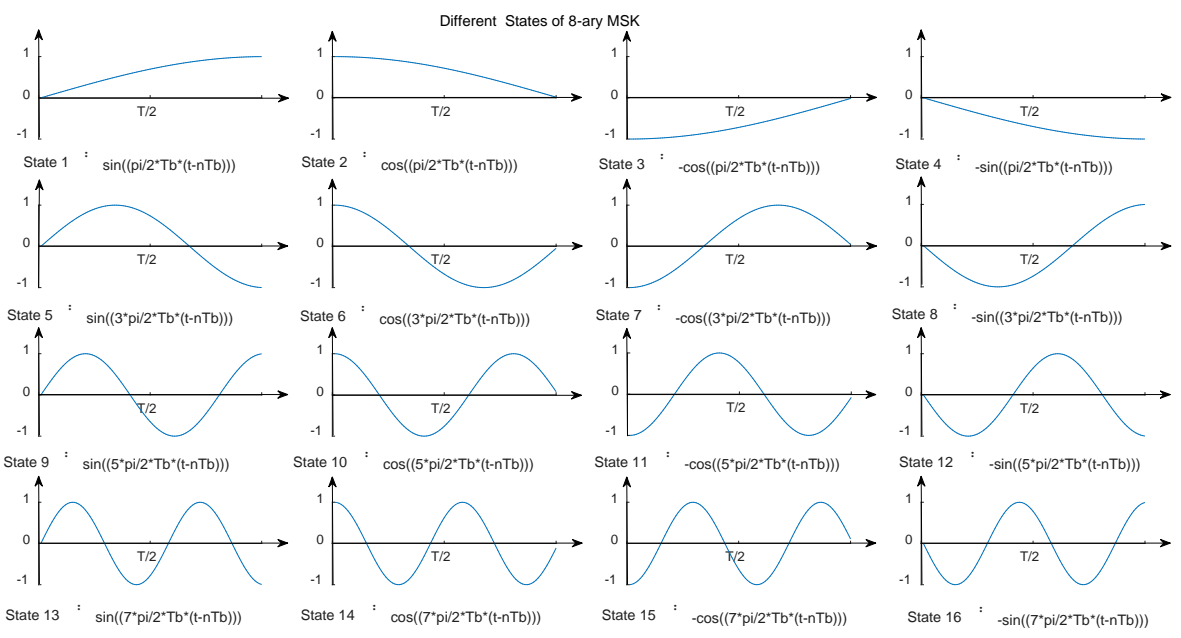

Fig. 2. Different states of 8-ary MSK

$$
\begin{aligned}
& P_{M}{ }^{1}=\frac{M}{2}\{\overbrace{\left(\begin{array}{ccccc}
A & A & \cdots & A & A \\
B & B & \cdots & B & B \\
\vdots & \vdots & \ddots & \vdots & \vdots \\
A & A & \cdots & A & A \\
B & B & \cdots & B & B
\end{array}\right)}^{M / 2} \\
& A=\left(\begin{array}{llll}
0 & a & 0 & 0 \\
b & 0 & 0 & b \\
b & 0 & 0 & b \\
0 & 0 & a & 0
\end{array}\right) B=\left(\begin{array}{cccc}
0 & 0 & a & 0 \\
b & 0 & 0 & b \\
b & 0 & 0 & b \\
0 & a & 0 & 0
\end{array}\right)
\end{aligned}
$$

The values of $a$ and $b$ depend on the number of $a$ or $b$ in each row, and the sum of probability each row should be equal to 1 .

c) In the case $m=2$ :

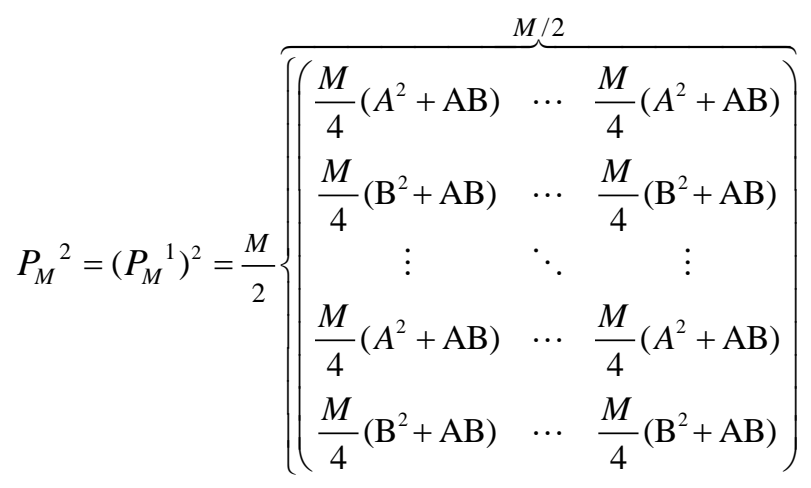




$$
A^{2}=B^{2}=A B=\left(\begin{array}{cccc}
a b & 0 & 0 & a b \\
0 & a b & a b & 0 \\
0 & a b & a b & 0 \\
a b & 0 & 0 & a b
\end{array}\right)
$$

In Fig. 2, take 8-ary MSK as an example. We can see that the first to fourth columns of the figure correspond to each of the four types of states in (3). The fact that state 1 ends magnitude one means that its next state must start withmagnitude one, the various states in the second column. It is easy to find the matrix of states arranged in this pattern, summarize to (6) and (7). To better understand matrix composition, examples of MSK, 4-ary MSK and 8-ary MSK are listed in Appendix 1.

Based on knowledge of stochastic processes, the autocorrelation function is given as follow expression from [21]:

$$
R(\tau)=E\left[X_{I}(t+\tau) X_{I}(t)\right]=R\left(m T+\tau^{\prime}\right)
$$

The above equation is the statistical average of the two random variables corresponding to the values taken by the stochastic process $X_{I}$ at two moments separated by $\tau$. Where $\tau=m T+\tau^{\prime}$ since $X_{I}$ is cyclostationary stochastic processes. In terms of the transition relationship between states and the corresponding transition probability, $R(\tau)$ can be expressed as:

$$
\begin{aligned}
& R(\tau)=R\left(m T+\tau^{\prime}\right)=\sum_{i=1}^{N} \sum_{j=1}^{N} P(\mathrm{i})\left[\mathrm{p}(\mathrm{j} \mid \mathrm{i}, \mathrm{m}) \mathrm{s}_{i j}\left(\mathrm{~T}-\tau^{\prime}\right)+\mathrm{p}(\mathrm{j} \mid \mathrm{i}, \mathrm{m}+1) \mathrm{s}_{j i}^{*}\left(\tau^{\prime}\right)\right] \\
& =\sum_{i=1}^{N} \sum_{j=1}^{N}\left\{P(\mathrm{i})\left[\mathrm{p}(\mathrm{j} \mid \mathrm{i}, \mathrm{m}) \mathrm{s}_{i j}\left(\mathrm{~T}-\tau^{\prime}\right)\right]+P(\mathrm{i})\left[\mathrm{p}(\mathrm{j} \mid \mathrm{i}, \mathrm{m}+1) \mathrm{s}_{j i}^{*}{ }^{*}\left(\tau^{\prime}\right)\right]\right\}
\end{aligned}
$$

where

$$
\begin{aligned}
& S_{i j}\left(T-\tau^{\prime}\right)=\frac{1}{T} \int_{0}^{T-\tau^{\prime}} S_{i}(u) s_{j}^{*}\left(u+\tau^{\prime}\right) d u \\
& S_{j i}^{*}\left(\tau^{\prime}\right)=\frac{1}{T} \int_{0}^{\tau^{\prime}} S_{j}^{*}(u) s_{i}\left(u+T-\tau^{\prime}\right) d u
\end{aligned}
$$

$P(\mathrm{i})$ as defined by (4), $s_{i}$ and $s_{j}$ are the signal corresponding to the phase that states at the time $i$ and $j$. The sign $*$ stands for conjugate. The following cases are divided into two parts and calculated where $m=0$ and $m=1$.

\subsection{Case 1: Where $0 \leq t \leq T$ and $\mathrm{m}=0$}

Based on the above Markov Chain Process Strategy, evaluate the autocorrelation functions of M-ary MSK. First, we calculate the autocorrelation function $R^{M}{ }_{m=0}(\tau)$ in a fixed time interval $[0, T]$. When $m=0$, equation (8) can be expressed as follows. 


$$
\begin{aligned}
R_{m=0}^{M}(\tau) & =R^{M}{ }_{m=0}\left(m T+\tau^{\prime}\right)=\sum_{i=1}^{N} \sum_{j=1}^{N} P(\mathrm{i})\left[\mathrm{p}(\mathrm{j} \mid \mathrm{i}, \mathrm{m}) \mathrm{s}_{i j}\left(\mathrm{~T}-\tau^{\prime}\right)+\mathrm{p}(\mathrm{j} \mid \mathrm{i}, \mathrm{m}+1) \mathrm{s}_{j i}{ }^{*}\left(\tau^{\prime}\right)\right] \\
& =\sum_{i}^{N} \sum_{j}^{N} P(\mathrm{i})\left[\mathrm{p}(\mathrm{j} \mid \mathrm{i}, \mathrm{m}) \mathrm{s}_{i j}\left(\mathrm{~T}-\tau^{\prime}\right)\right]+\sum_{i}^{N} \sum_{j}^{N} P(i)\left[p(j \mid i, m+1) s_{j i}{ }^{*}\left(\tau^{\prime}\right)\right] \\
& =R 1^{M}{ }_{m=0}(\tau)+R 2^{M}{ }_{m=0}(\tau)
\end{aligned}
$$

To facilitate the operation, the autocorrelation function $R^{M}{ }_{m=0}(\tau)$ can be divided into the sum of two parameters $R 1^{M}{ }_{m=0}(\tau)$ and $R 2^{M}{ }_{m=0}(\tau)$ for the procedure. Before that, since the excessive number of terms in the expansion formula, patterns can only be found in the calculation process in 4-ary and 8-ary first.

$$
\begin{aligned}
R_{m=0}^{M}(\tau) & =\frac{1}{2 M}\left\{2 s_{11}+2 s_{22}+2 s_{55}+2 s_{66}+\frac{1}{2} s_{21}{ }^{*}-\frac{1}{2} s_{25}{ }^{*}+\frac{1}{2} s_{61}{ }^{*}-\frac{1}{2} s_{65}{ }^{*}\right\} \\
& +\frac{1}{2 M}\left\{\frac{1}{2} s_{10,1}{ }^{*}-\frac{1}{2} s_{10,5}{ }^{*}+\frac{1}{2}{s_{14,1}}^{*}-\frac{1}{2} s_{14,5}{ }^{*}\right\} \\
& +\frac{1}{2 M}\left\{2 s_{99}+2 s_{10,10}+2 s_{13,13}+2 s_{14,14}+\frac{1}{2} s_{29}{ }^{*}-\frac{1}{2} s_{2,13}{ }^{*}+\frac{1}{2} s_{6,9}{ }^{*}-\frac{1}{2} s_{6,13}{ }^{*}\right\} \\
& +\frac{1}{2 M}\left\{\frac{1}{2} s_{10,9}{ }^{*}-\frac{1}{2} s_{10,13}{ }^{*}+\frac{1}{2} s_{14,9}{ }^{*}-\frac{1}{2} s_{14,13}{ }^{*}\right\} \\
& +\frac{1}{2 M}\{\cdots\}
\end{aligned}
$$

The partial expansion of $R^{M}{ }_{m=0}(\tau)$ is shown above, with the first row being the result of a 4-ary calculation and the first four rows being the result of an 8-ary calculation, thus concluding that:

$$
\begin{aligned}
R 1_{m=0}^{M}(\tau) & =\sum_{i}^{N} \sum_{j}^{N} P(\mathrm{i})\left[\mathrm{p}(\mathrm{j} \mid \mathrm{i}, \mathrm{m}) \mathrm{s}_{i j}\left(\mathrm{~T}-\tau^{\prime}\right)\right]=\frac{1}{M}\left[s_{11}+s_{22}+s_{33}+\cdots+s_{M M}\right] \\
& =\frac{2}{M}\left[\frac{1}{\mathrm{~T}} \int_{0}^{\mathrm{T}-\tau^{\prime}} \sin \frac{n \pi}{2 \mathrm{~T}} u \sin \frac{n \pi}{2 \mathrm{~T}}\left(\mathrm{u}+\tau^{\prime}\right) \mathrm{du}\right] \\
& =\frac{2}{M}\left[\frac{1}{2}\left(1-\frac{\tau^{\prime}}{\mathrm{T}}\right) \cos \frac{\pi}{2 \mathrm{~T}} \tau^{\prime}+\frac{1}{2}\left(1-\frac{\tau^{\prime}}{\mathrm{T}}\right) \cos \frac{3 \pi}{2 \mathrm{~T}} \tau^{\prime}+\cdots+\frac{1}{2}\left(1-\frac{\tau^{\prime}}{\mathrm{T}}\right) \cos \frac{n \pi}{2 \mathrm{~T}} \tau^{\prime}\right] \\
& =\frac{2}{M} \sum_{n=1}^{M-1} \frac{1}{2}\left(1-\frac{\tau^{\prime}}{T}\right) \cos \frac{n \pi \tau^{\prime}}{2 T}, \quad n=1,3,5 \ldots
\end{aligned}
$$

The result of integration is different for different $n, m$ terms with various positive and negative signs. $R 2^{M}{ }_{m=0}(\tau)$ is the result of the accumulation of the terms. What's more, we found that differences in the initial phases of cos and sin within the integral lead to different generalizations of the integral result. Therefore, the $R 2^{M}{ }_{m=0}(\tau)$ is divided into two terms according to whether the $n, m$ is the same or not. $\sum_{n=1}^{M=1}$ denotes an odd summation of $n$ from 1 to $(M-1)$. 


$$
\begin{aligned}
& R 2^{M}{ }_{m=0}(\tau)=\sum_{i}^{N} \sum_{j}^{N} P(i)\left[p(j \mid i, m+1) s_{j i}{ }^{*}\left(\tau^{\prime}\right)\right]=R 2 \_\operatorname{same}^{M}{ }_{m=0}(\tau)+R 2 \_\operatorname{dif}^{M}{ }_{m=0}(\tau) \\
& =\frac{2}{M^{2}} *\left\{\begin{array}{l}
(-1)^{(n+1) / 2} \sum_{n}^{M-1}\left\{\frac{1}{T} \int_{0}^{\tau^{\prime}} \cos \frac{n \pi}{2 T} u \sin \frac{n \pi}{2 T}\left(u+T-\tau^{\prime}\right) d u\right\}, n=1,3,5 \ldots \\
(-1)^{(m+1) / 2} \sum_{n=1}^{M-1} \sum_{m=1}^{M-1} \frac{1}{T} \int_{0}^{\tau^{\prime}} \cos \frac{n \pi}{2 T} u \sin \frac{m \pi}{2 T}\left(u+T-\tau^{\prime}\right) d u, n \neq m \text { and } n, m=1,3,5 \ldots
\end{array}\right. \\
& =\frac{2}{M^{2}} *\left\{\begin{array}{l}
(-1)^{(n+3) / 2} \sum_{n=1}^{M-1}\left\{\frac{1}{2} \frac{\tau^{\prime}}{T} \sin \frac{\pi\left(T-\tau^{\prime}\right) n}{2 T}+\frac{\sin \left(\frac{1}{2} \pi n\right) \sin \frac{\pi \tau^{\prime} n}{2 T}}{\pi n}\right\}, n=1,3,5 \ldots \\
(-1)^{(m+1) / 2} \sum_{n=1}^{M-1} \sum_{m=1}^{M-1} 2 * \frac{n\left[\sin \left(\frac{1}{2} \pi m\right) \sin \frac{\pi \tau^{\prime} n}{2 T}\right]-m\left[\cos \frac{\pi\left(T-\tau^{\prime}\right) m}{2 T}\right]}{\pi\left[m^{2}-n^{2}\right]}, n \neq m \text { and } n, m=1,3,5 \ldots
\end{array}\right.
\end{aligned}
$$

\subsection{Case2: Where $\mathrm{T} \leq \mathrm{t} \leq 2 \mathrm{~T}$ and $\mathrm{m}=1$}

The calculation process is similar to that of Case $1 . R_{m=1}^{M}(\tau)$ is divided into $R 1_{-} \operatorname{same}^{M}{ }_{m=1}(\tau)$ and $R 1_{-} \operatorname{dif}^{M}{ }_{m=1}(\tau)$ for the summation calculation.

$$
\begin{aligned}
R^{M}{ }_{m=1}(\tau) & =R^{M}{ }_{m=1}\left(m T+\tau^{\prime}\right)=\sum_{i=1}^{N} \sum_{j=1}^{N} P(\mathrm{i})\left[\mathrm{p}(\mathrm{j} \mid \mathrm{i}, \mathrm{m}) \mathrm{s}_{i j}\left(\mathrm{~T}-\tau^{\prime}\right)+\mathrm{p}(\mathrm{j} \mid \mathrm{i}, \mathrm{m}+1) \mathrm{s}_{j i}^{*}\left(\tau^{\prime}\right)\right] \\
& =\sum_{i=1}^{N} \sum_{j=1}^{N}\left\{P(\mathrm{i})\left[\mathrm{p}(\mathrm{j} \mid \mathrm{i}, \mathrm{m}) \mathrm{s}_{i j}\left(\mathrm{~T}-\tau^{\prime}\right)\right]+P(\mathrm{i})\left[\mathrm{p}(\mathrm{j} \mid \mathrm{i}, \mathrm{m}+1) \mathrm{s}_{j i}{ }^{*}\left(\tau^{\prime}\right)\right]\right\} \\
& =\sum_{i=1}^{N} \sum_{j=1}^{N} P(\mathrm{i})\left[\mathrm{p}(\mathrm{j} \mid \mathrm{i}, \mathrm{m}) \mathrm{s}_{i j}\left(\mathrm{~T}-\tau^{\prime}\right)\right]
\end{aligned}
$$

Since the high symmetry and repetition of the matrix, the $P(\mathrm{i})\left[\mathrm{p}(\mathrm{j} \mid \mathrm{i}, \mathrm{m}+1) \mathrm{s}_{\mathrm{j}^{*}}{ }^{*}\left(\tau^{\prime}\right)\right]$ term is eliminated during the calculation. Summing up the items gives:

$$
\begin{aligned}
& R^{M}{ }_{m=1}(\tau)=R 1^{M}{ }_{m=1}(\tau) \\
& =R 1_{-} \text {same }^{M}{ }_{m=1}(\tau)+R 1_{-} \operatorname{dif}^{M}{ }_{m=1}(\tau) \\
& =\frac{2}{M^{2}} *\left\{\begin{array}{l}
(-1)^{(n+1) / 2} \sum_{n}^{M}\left\{\frac{1}{2}\left(1-\frac{\tau^{\prime}}{T}\right) \sin \frac{\pi \tau^{\prime} n}{2 T}-\frac{\sin \left(\frac{1}{2} \pi n\right) \sin \frac{\pi\left(T-\tau^{\prime}\right) n}{2 T}}{n \pi}\right\}, \mathrm{n}=1,3,5 \ldots \\
(-1)^{(n+3) / 2} \sum_{n}^{M} \sum_{m}^{M} \frac{(n-m) \cos \frac{\pi\left[T m+\left(T-\tau^{\prime}\right) n\right]}{2 T}-2 n \cos \frac{\pi \tau^{\prime} m}{2 T}+(m+n) \cos \frac{\pi\left(T m+\left(\tau^{\prime}-T\right) n\right)}{2 T}, n \neq m \text { and } n, m=1,3,5 \ldots}{\left(m^{2}-n^{2}\right) \pi}
\end{array}\right.
\end{aligned}
$$

Bring $\tau^{\prime}=\left\{\begin{array}{lll}\tau & m=0 & (0 \leq \tau \leq \mathrm{T}) \\ \tau-\mathrm{T} & m=1 & (\mathrm{~T} \leq \tau \leq 2 \mathrm{~T})\end{array}\right.$ into (10) to get the autocorrelation function, so the final M-ary MSK expression is:

$$
R^{M}(\tau)=R_{m=0}^{M}(\tau)+R_{m=1}^{M}(\tau)=R 1^{M}{ }_{m=0}(\tau)+R 2^{M}{ }_{m=0}(\tau)+R_{m=1}^{M}(\tau)
$$

Where $T$ is a time interval. Because of the symmetry of the correlation function, the expressions in $-2 T \leq t \leq-T$ and $-T \leq t \leq 0$ are equal to equations $T \leq t \leq 2 T$ and $0 \leq t \leq T$, 
respectively.

\section{PSD function}

Once the autocorrelation function $R^{M}(\tau)$ in section 3 has been evaluated. The PSD function can be derived directly by performing a Fourier transform on the autocorrelation function. Here is the derivation process:

$$
\begin{aligned}
S^{M} & =F\left\{R^{M}(\tau)\right\}=\int_{-\infty}^{\infty} R^{M}(\tau) e^{-j \omega \tau} d \tau=2 \int_{-\infty}^{\infty} R^{M}(\tau) \cos 2 \pi f \tau d \tau \\
& =2 \int_{-\infty}^{\infty} R^{M}{ }_{m=0}(\tau) \cos 2 \pi f \tau d \tau+2 \int_{-\infty}^{\infty} R^{M}{ }_{m=1}(\tau) \cos 2 \pi f \tau d \tau \\
& =S^{M}{ }_{m=0}+S^{M}{ }_{m=1}
\end{aligned}
$$

Based on (12), the PSD function can easily be derived by direct calculation, and the detailed derivations are moved to Appendix 2. The following cases are solved for $S^{M}{ }_{m=0}$ and $S^{M}{ }_{m=1}$.

\subsection{Case 1: Where $0 \leq \mathrm{t} \leq \mathrm{T}$ and $\mathrm{m}=0, S_{m=0}^{M}$}

$$
\begin{aligned}
S^{M}{ }_{m=0}(f) & =\sum_{i=1}^{N} \sum_{j=1}^{N} \int_{-\infty}^{\infty} 2 P(\mathrm{i})\left[\mathrm{p}(\mathrm{j} \mid \mathrm{i}, \mathrm{m}) \mathrm{s}_{i j}\left(\mathrm{~T}-\tau^{\prime}\right)\right] \cos 2 \pi f \tau d \tau+\sum_{i=1}^{N} \sum_{j=1}^{N} \int_{-\infty}^{\infty} 2 P(\mathrm{i})\left[\mathrm{p}(\mathrm{j} \mid \mathrm{i}, \mathrm{m}+1) \mathrm{s}_{j i}{ }^{*}\left(\tau^{\prime}\right)\right] \cos 2 \pi f \tau d \tau \\
= & S 1^{M}{ }_{m=0}(f)+S 2 \_\operatorname{same}^{M}{ }_{m=0}(f)+S 2_{-} d i f^{M}{ }_{m=0}(f) \\
S 1^{M}{ }_{m=0}(f) & =T\left\{\frac{\sin [\varphi(n)+\omega(f)]}{2[\varphi(n)+\omega(f)]}+\frac{\sin [\varphi(n)-\omega(f)]}{2[\varphi(n)-\omega(f)]}-\frac{\cos [\varphi(n)-\omega(f)]+[\varphi(n)-\omega(f)] \sin [\varphi(n)-\omega(f)]-1}{2[\varphi(n)-\omega(f)]^{2}}\right. \\
& \left.-\frac{\cos [\varphi(n)+\omega(f)]+[\varphi(n)+\omega(f)] \sin [\varphi(n)+\omega(f)]-1}{2[\varphi(n)+\omega(f)]^{2}}\right\}
\end{aligned}
$$

$$
\begin{aligned}
S 2 \_\operatorname{same}_{m=0}(f) & =4 T \frac{\varphi(n)\left[\cos \omega(f) * S_{2}+8 \omega(f) \sin \omega(f)\right]-\sin \varphi(n) * S_{1}(n)}{S_{2}^{2}(n)} \\
& -4 T \frac{\sin \varphi(n)[\omega(f) \sin \omega(f) \sin \varphi(n)+\varphi(n) \cos \omega(f) \cos \varphi(n)-\varphi(n)]}{\varphi(n) S_{2}(n)}
\end{aligned}
$$

$$
\begin{aligned}
S 2 \_d i f^{M}{ }_{m=0}(f) & =8 T \frac{\varphi(n) \sin \varphi(m) *[\omega(f) \sin \omega(f) \sin \varphi(n)+\varphi(n) \cos \omega(f) \cos \varphi(n)-\varphi(n)]}{[\varphi(n)-\varphi(m)][\varphi(n)+\varphi(m)] S_{2}(n)} \\
& +8 T \varphi(m) \frac{\varphi(m) \sin \varphi(m)-\omega(f) \sin \omega(f)}{[\varphi(n)-\varphi(m)][\varphi(n)+\varphi(m)] S_{2}(m)}
\end{aligned}
$$

4.2 Case 2: Where $\mathrm{T} \leq \mathrm{t} \leq 2 \mathrm{~T}$ and $\mathrm{m}=1, S_{m=1}^{M}$

$$
\begin{aligned}
S^{M}{ }_{m=1} & =\sum_{i=1}^{N} \sum_{j=1}^{N} \int_{-\infty}^{\infty} 2 P(\mathrm{i})\left[\mathrm{p}(\mathrm{j} \mid \mathrm{i}, \mathrm{m}) \mathrm{s}_{i j}\left(\mathrm{~T}-\tau^{\prime}\right)\right] \cos 2 \pi f \tau d \tau \\
& =S 1 \_ \text {same }^{M}{ }_{m=1}(f)+S 1_{-} d i f^{M}{ }_{m=1}(f)
\end{aligned}
$$


$S^{M}{ }_{m=1}$ is divided into $S 1_{-} \operatorname{same}_{m=1}^{M}(f)$ and $S 1_{-} \operatorname{dif}^{M}{ }_{m=1}(f)$ for the summation calculation.

$$
\begin{aligned}
S 1_{-} \text {same }_{m=1}(f) & =-2 T \frac{2[\varphi(n)+\omega(f)] \cos [\varphi(n)-2 \omega(f)]+2[\varphi(n)-\omega(f)] \cos [\varphi(n)+2 \omega(f)]-4 \varphi(n) \cos \omega(f)}{S_{2}(n)} \\
& +2 T\left\{\frac{2[\varphi(n)-\omega(f)] \cos [\varphi(n)-2 \omega(f)]-\sin [\varphi(n)-2 \omega(f)]}{4[\varphi(n)-\omega(f)]^{2}}\right. \\
& \left.+\frac{[\varphi(n)+\omega(f)] \cos [\varphi(n)+2 \omega(f)]-\sin [\varphi(n)+2 \omega(f)]}{4[\varphi(n)+\omega(f)]^{2}}-\frac{2 \varphi(n)\left[S_{2} \cos \omega(f)+8 \omega(f) \sin \omega(f)\right]}{S_{2}^{2}(n)}\right\} \\
& +2 T \sin \varphi(n)\left[\frac{\cos [\varphi(n)-\omega(f)]+\cos [\varphi(n)+\omega(f)]-2 \cos 2 \omega(f)}{S_{2}(n)}-\frac{2 \omega(f) \sin \omega(f) \sin \varphi(n)}{\varphi(n) S_{2}(n)}\right]
\end{aligned}
$$

$$
\begin{aligned}
S 1 \_d i f^{M}{ }_{m=1}(f) & =\frac{T}{2}\left\{\frac{\sin [\varphi(m)+2 \omega(f)]-\sin [\varphi(n)+\varphi(m)+\omega(f)]}{[\varphi(n)+\varphi(m)][\varphi(n)-\omega(f)]}-\frac{\sin [2 \omega(f)-\varphi(m)]+\sin [\varphi(n)+\varphi(m)-\omega(f)]}{[\varphi(n)+\varphi(m)][\varphi(n)+\omega(f)]}\right\} \\
& -T \varphi(n) \frac{[\varphi(m)-\omega(f)] \sin [\varphi(m)+2 \omega(f)]+[\varphi(m)+\omega(f)] \sin [\varphi(m)-2 \omega(f)]+2 \omega(f) \sin \omega(f)}{\left[\varphi^{2}(m)-\varphi^{2}(n)\right]\left[\varphi^{2}(m)-\omega^{2}(f)\right]} \\
& +T\left\{\frac{\sin [\varphi(m)+2 \omega(f)]-\sin [\varphi(m)-\varphi(n)+\omega(f)]}{2[\varphi(m)-\varphi(n)][\varphi(n)+\omega(f)]}-\frac{\sin [\varphi(m)-\varphi(n)-\omega(f)]-\sin [\varphi(m)-2 \omega(f)]}{2[\varphi(m)-\varphi(n)][\varphi(n)-\omega(f)]}\right\}
\end{aligned}
$$

Where

$$
\begin{aligned}
& S_{1}(n)=4\left[\varphi^{2}(n)+\omega^{2}(f)\right]=\pi^{2}\left(n^{2}+16 f^{2} T^{2}\right) \\
& S_{2}(n)=4\left[\varphi^{2}(n)-\omega^{2}(f)\right]=\pi^{2}\left(n^{2}-16 f^{2} T^{2}\right) \\
& S_{1}(m)=4\left[\varphi^{2}(m)+\omega^{2}(f)\right]=\pi^{2}\left(m^{2}+16 f^{2} T^{2}\right) \\
& S_{2}(m)=4\left[\varphi^{2}(m)-\omega^{2}(f)\right]=\pi^{2}\left(m^{2}-16 f^{2} T^{2}\right) \\
& \omega(f)=2 \pi f T \quad \varphi(m)=m \pi / 2 \quad \varphi(n)=n \pi / 2 \quad n, m=1,3,5 \ldots
\end{aligned}
$$

\section{Simulation for Verification and Analysis}

The expression results are compared with those signal simulations using MATLAB to verify the accuracy of autocorrelation functions and PSDs. The result is shown in Fig. 3, Fig. 4, Fig. 5, and Fig. 6. We set simulation parameters as the length of the simulated input signal $n=10^{5}$, code element time interval $T=0.1 \mathrm{~s}$, bit time interval $T_{b}=T / \log _{2} M$, sampling frequency $f_{s}=10^{5} \mathrm{~Hz}$.

\section{1 autocorrelation function of M-ary MSK}

In Fig. 3, it can be concluded that the larger the value of $M$, the more the autocorrelation function is concentrated from $-T$ to $T$. As a result, the amplitude between $T$ to $2 T$ and $-2 T$ to $-T$ is getting lower, closer to 0 . We can still conclude that there is no discrepancy between the theoretical autocorrelation function expressions in (11) and obtained from the 
simulation. And to understand the subtle differences between the theoretical expressions and simulation, Fig. 4 is drawn to compare the details of the octal signal as an example.

In Fig. 4, we can see that the overall shape has not changed in most of the exact matches, but there are subtle differences in the magnitude. Although this property is verified with little doubt, one can reasonably conclude that both two have approximately the same. In the simulation process, it was found that as the length of the simulated input signal $n$ increases, the difference between the two curves will become lower, and the simulated curve will gradually approach the theoretical curve. So we believe that the reason for the slight difference in the curves is the experimental error.
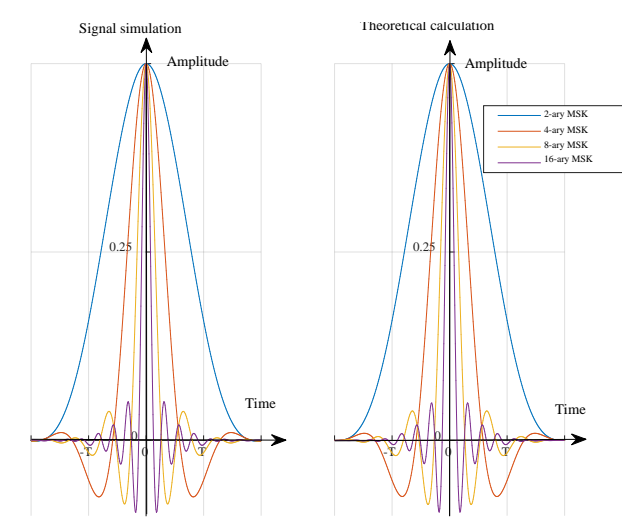

Fig. 3. Comparison of M-ary MSK autocorrelation function, $\mathrm{M}=2,4,8,16$

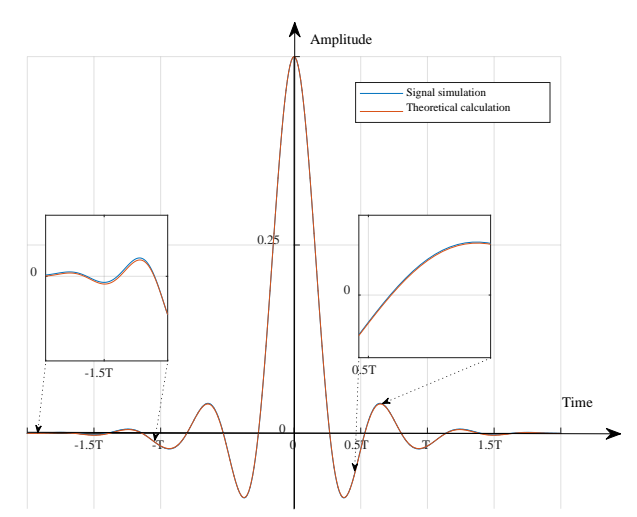

Fig. 4. Comparison of theoretical expression curve and signal simulation curve of 8-ary MSK autocorrelation function

\subsection{Power spectral-density function}

As 8-ary MSK autocorrelation function, we take the spectrum of 4-ary and 8-ary MSK as an example to verify the accuracy. Fig. 5 and Fig. $\mathbf{6}$ show that the simulation results match highly with the theoretical derivation in the amplitude of the main lobe and the first side lobe, after which distortion occurs because of the cut-off effect of the Fourier transform of the signal simulation. 


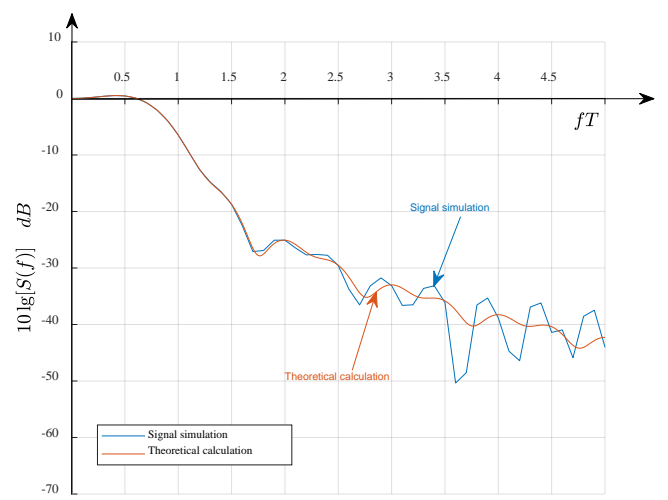

Fig. 5. Theoretical expression curve and signal simulation curve of 4-ary MSK power spectraldensity function

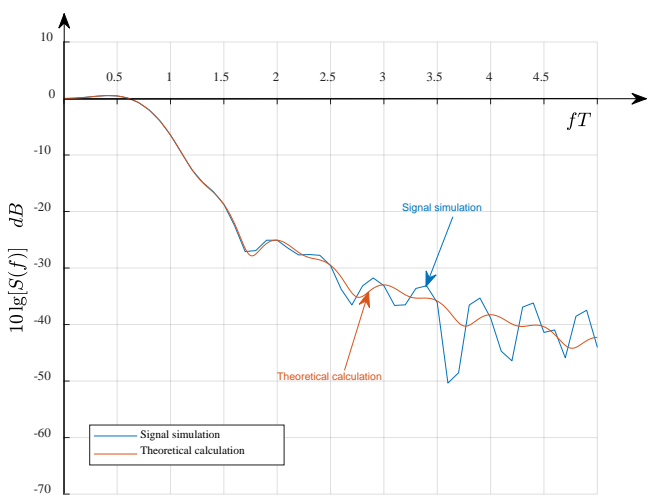

Fig. 6. Comparison of theoretical expression curve and signal simulation curve of 16-ary MSK power spectral-density function

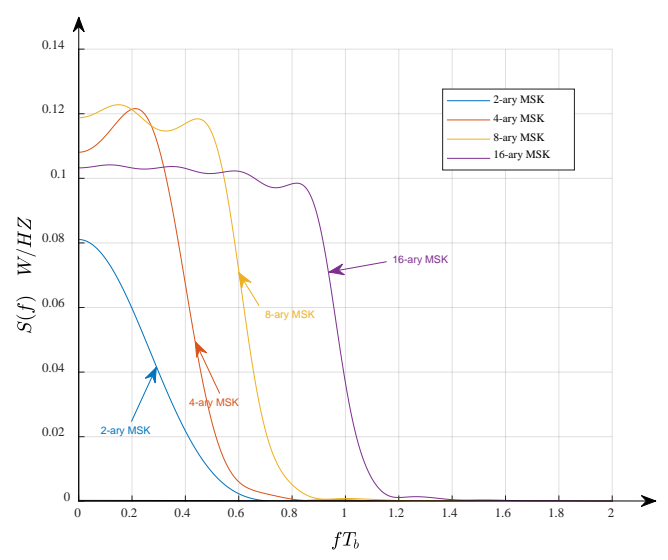

Fig. 7. Normalised PSDs of M-ary MSK,M=2,4,8,16

The number of bits increases within a code element interval. This leads to the dispersion of energy in the spectrum. So it is necessary to normalize the energy to one bit when making the comparison. The horizontal coordinate is set to $f T_{b}$.

The PSD for $M=2,4,8,16$ is shown in Fig. 7 . We can see that at $M=4$ and 8 the amplitude spectrum becomes more concentrated, and the signal intensity increase more. As well known, the PSD of higher-level $M$ rolls off much quicker than that $M=2$.

When $M=16$, the top of the lobe is flatter so that the bandwidth can be better utilized and the transmission efficiency becomes improved, but $\mathrm{h}$ is fixed equal to 0.5. The spectral main lobe bandwidth of M-ary MSK becomes wider substantially with the number of levels up. 

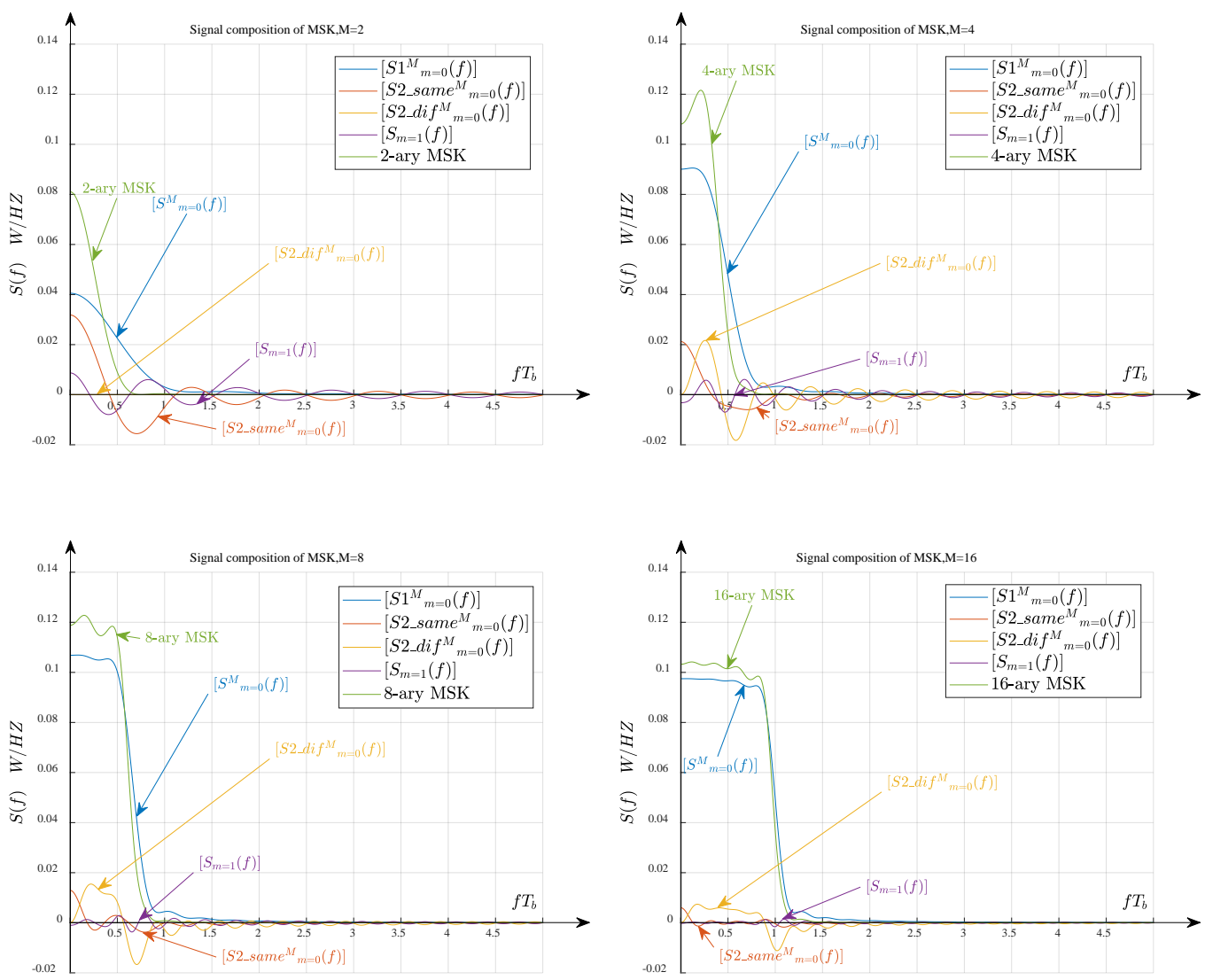

Fig. 9. Signal composition of $M S K, M=2,4,8,16$

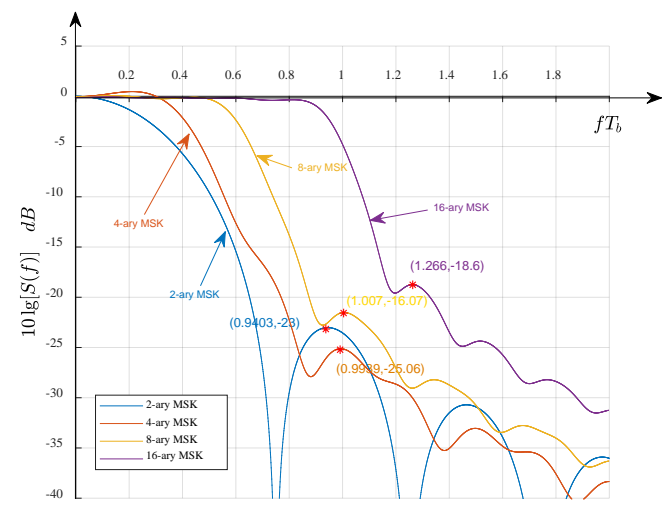

Fig. 8. Logarithmic form of normalized power spectral densities of M-ary MSK, M=2,4,8,16

In Fig. 8, the logarithmic form of the spectrum function can be seen. It is worth mentioning that the first side lobe of 4-ary MSK is lower than that of MSK, and the main lobe is higher, and the energy is more concentrated. 


\subsection{Spectrum composition}

Fig. 9 shows the composition of the spectrum of M-ary MSK where $M=2,4,8,16$. Because for the case of $m=1$, the spectrum with many higher-order components whose amplitude becomes extremely low and the proportion of the overall, so it is considered a variable for comprehensive analysis. For the case of $\mathrm{m}=0$, the proportion is more significant, and the subdivision is more detailed.

As far as those figures, we can provide an analysis of the composition of the spectrum in detail. The first one we can see is $S 1^{M}{ }_{m=0}(f)$ contributed the majority of the spectrum and is more pronounced when the level $\mathrm{M}$ increases. Second, $S 2_{-} d i f^{M}{ }_{m=0}(f)$ determine the shape of the top of the spectrum waveform, and the fluctuations become smaller with the increase of $\mathrm{M}$, which is why the top of the lobe gets flattered within a specific bandwidth.

\subsection{Fractional out-of-band power}

It is also helpful to show the out-of-band power with M-ary MSK. It defines as [10]:

$$
P_{o b}=1-\left\{\int_{-B}^{+B} S(f) d f / \int_{-\infty}^{+\infty} S(f) d f\right\}
$$

In Fig. 10, $P_{o b}$ is plotted against $B T_{b}$ for M-ary MSK where $M=2,4,8,16$. We can see 4-ary MSK has less out-of-band power compared to 2-ary MSK when $B T_{b}>0.71$, while $B T_{b}>0.84,8$-ary MSK has less than 2-ary MSK but upper than 4-ary MSK.

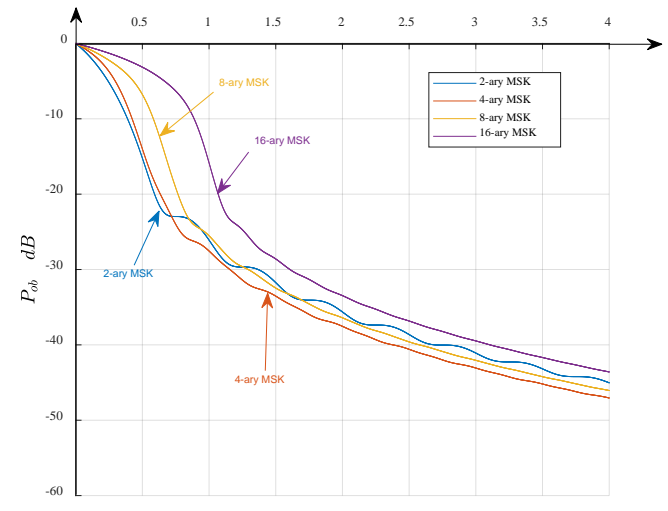

Fig. 10. Fractional out-of-band power of MSK, $M=2,4,8,16$

\section{Optimum Maximum Likelihood Coherent Detection}

The M-ary MSK detection in this paper uses coherent demodulation with maximum likelihood probability. In a code-time $T$, the phase state will increase or decrease $1 / 2 \pi D_{d}$ with the input data $d_{i}$ based on the starting phase $\theta$. For the modulation index $\mathrm{h}=1 / 2$, there are $2 * 2 \pi /(1 / 2 \pi)=8$ possibilities for the phase at the beginning of a code element. Because of the symmetry of the cosine function, the beginning phase states are reduced by half to four. 
For each beginning phase, the phase change can be generated according to the input symbols, so there are $k_{\max }=4 M$ theoretical variations, denote as $\Phi_{k}$. Then the waveform at the time $i$ of each received codeword can be correlated with:

$$
\begin{gathered}
l_{1}=\int_{(i-1) T}^{i T} \cos \Phi_{1}(t) * r(t) d t \\
l_{2}=\int_{(i-1) T}^{i T} \cos \Phi_{2}(t)^{*} r(t) d t \\
\vdots \\
l_{4 M-1}=\int_{(i-1) T}^{i T} \cos \Phi_{4 M-1}(t)^{*} r(t) d t \\
l_{4 M}=\int_{(i-1) T}^{i T} \cos \Phi_{4 M}(t) * r(t) d t
\end{gathered}
$$

Where $l_{K}=\max \left(l_{1}, l_{2}, \cdots, l_{4 M-1}, l_{4 M}\right)$.

We get the maximum value $l_{K}$. The $\cos \Phi_{K}(t)$ is chosen as the best reception for this waveform at the time $i$. We can also reduce the BER of the received signal with coherent demodulation by increasing the inherent memory L. In this case, $\Phi_{k}$ expressed as $\Phi^{L}{ }_{k}$, the optimal receive memory inherent $L$ corresponds to the observation time of the code element while satisfying the condition of phase continuity. The numbers of all possibilities of length $\mathrm{L}, k_{\max }$ is then increased to $4 M^{L}$.

$$
\begin{gathered}
I_{1}^{L}=\int_{(i-1) T}^{(i+L-1) T} \cos \Phi^{L}{ }_{1}(t) * r(t) d t \\
I^{L}{ }_{2}=\int_{(i-1) T}^{(i+L-1) T} \cos \Phi^{L}{ }_{2}(t) * r(t) d t \\
\vdots \\
I_{4 M^{2}-1}^{L}=\int_{(i-1) T}^{(i+L-1) T} \cos \Phi^{L}{ }_{4 M^{2}-1}(t) * r(t) d t \\
I_{4 M^{2}}^{L}=\int_{(i-1) T}^{(i+L-1) T} \cos \Phi_{4 M^{2}}^{L}(t) * r(t) d t \\
I^{L}=\max \left(I^{L}{ }_{1}^{L}, I^{L}{ }_{2}, \cdots, I^{L}{ }_{4 M^{L}-1}, l^{L}{ }_{4 M^{L}}\right) ;
\end{gathered}
$$

Fig. 11 shows the Receiving process. The diagram on the left shows the reception of the first code element. We correlate the received signal code elements numbered T1 to T(L) with $\cos \Phi_{1}^{L}, \cos \Phi_{2}^{L}, \ldots, \cos \Phi_{4 M^{L}-1}^{L} \cos \Phi_{4 M^{L}}^{L}$ to get $l_{1}^{L}, l_{2}^{L}, \ldots, l_{4 M^{L}-1}^{L}, l_{4 M^{L}}^{L}$ following (14). The waveform $\cos \Phi_{K}^{L}(t)$ corresponding to the largest $l_{K}^{L}$ is selected as the best reception for this length $L$ signal. Choose only the first one as the decoder for this reception afterward.

The right part of Fig. 11 shows the reception of the second code element, the range of detection is shifted to the right by one code element in length, and the matching method is similar to that of the first code element and continues. 

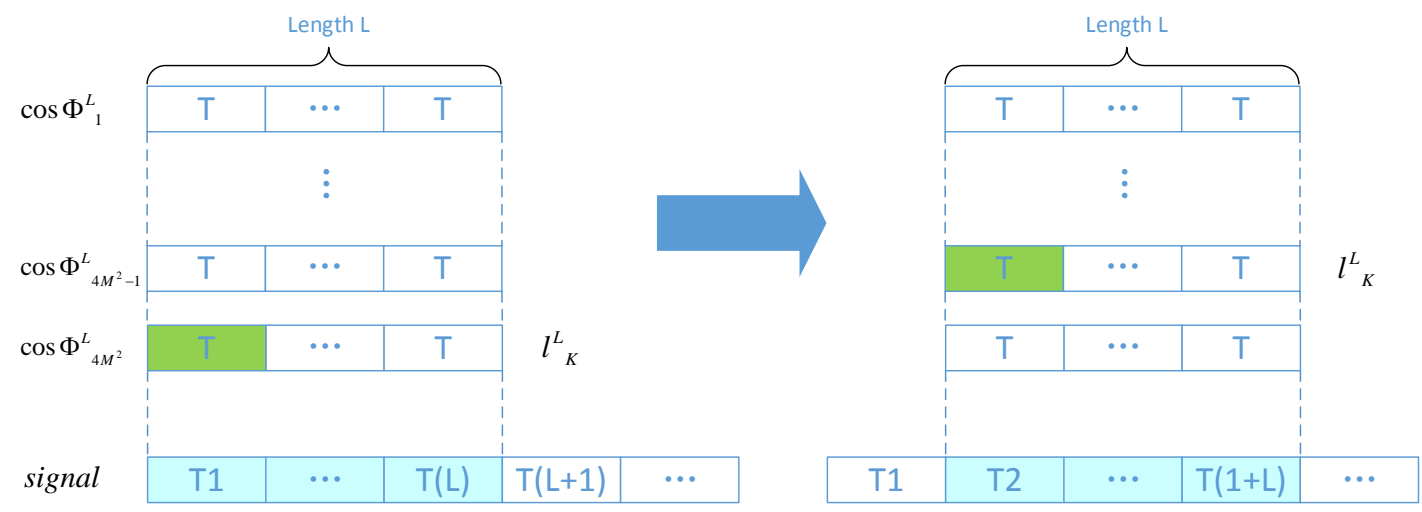

Fig. 11. Length L best match schematic
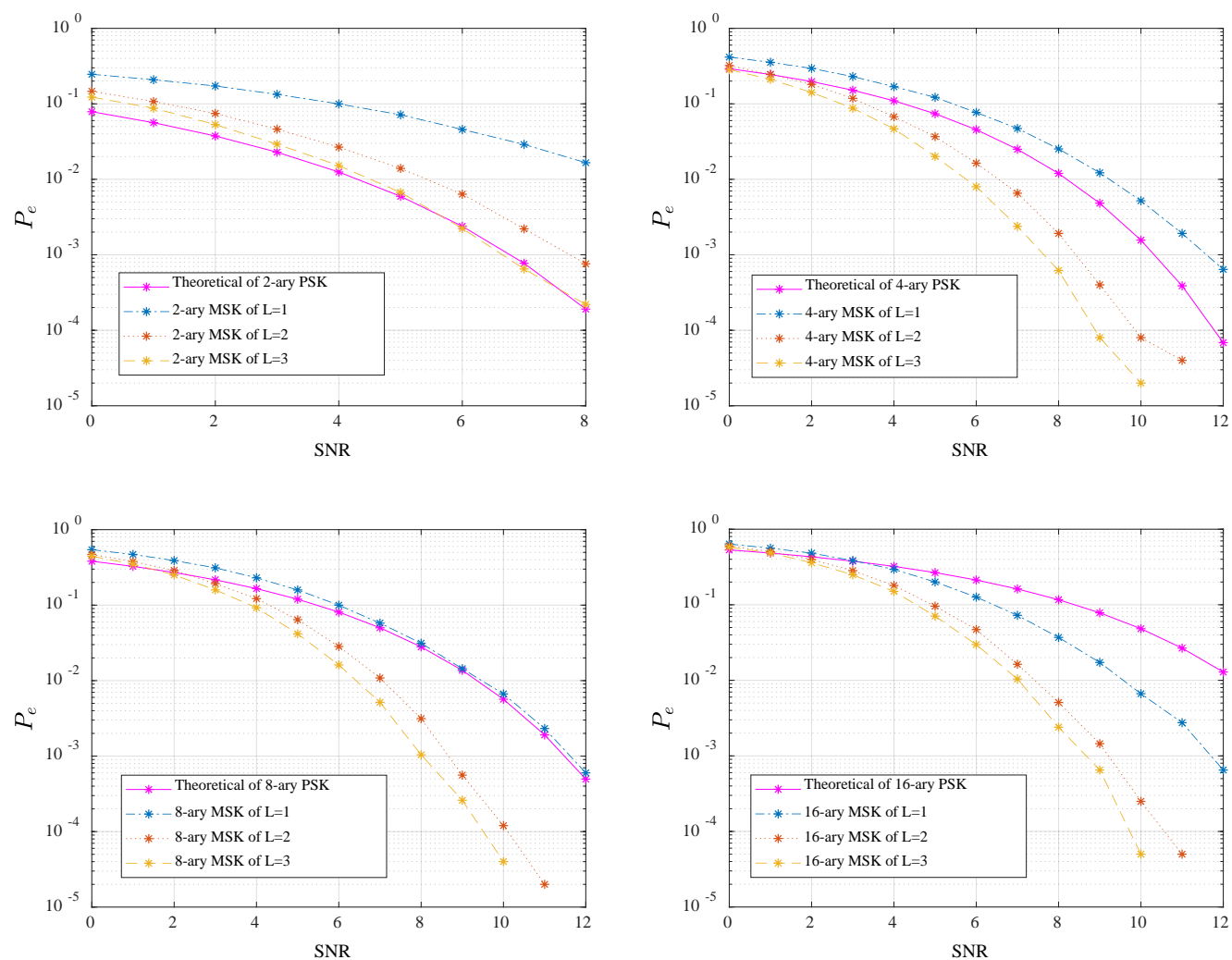

Fig. 12. Probability of symbol error for M-ary MSK, $M=2,4,8,16$

We set: $h=1 / 2, S N R=E_{b} /\left(N_{0} \log _{2} M\right)$

Sampling frequency $f s=20000$

Symbol duration $T=0.001$

Number of input symbols $n=100000$

The theoretical BER of M-PSK is as follows: 
BPSK theoretical BER: $P_{e}=1-\frac{1}{2} \operatorname{erfc} \sqrt{r}$

QPSK theoretical BER: $P_{e}=1-\left[1-\frac{1}{2} \operatorname{erfc} \sqrt{r / 2}\right]^{2}$

MPSK theoretical BER: $P_{e} \approx \operatorname{erfc}\left(\sqrt{2 r} \sin \frac{\pi}{2 M}\right)$

The BER curves of 2-ary MSK, 4-ary MSK, and 8-ary MSK are obtained by MATLAB simulation, respectively in Fig. 12. When $L=1$, compared with theoretical M-ary PSK under each of the same M, 2-ary MSK OMLCD only approximates theoretical values. But for M > 2, the overall BER of the correlate reception can be better than the theoretical M-ary PSK, and the larger $\mathrm{M}$, the better it is. This reflects the relative superiority of OMLCD compared to theoretical PSK when arys increase.

To add optimal memory inherent $\mathrm{L}$, there is no doubt that it will significantly improve BER. Those four MSK average lift of about $4 \mathrm{~dB}$ when $\mathrm{L}=3$ compared with $\mathrm{L}=1$ at high SNR, for the case of increasing L from 1 to 2 BER improve about $3 \mathrm{~dB}$. Specifically, 4-ary MSK with $\mathrm{L}=3$ can promote theoretical QPSK BER by $3.1 \mathrm{~dB}$, and the coherent 16-ary MSK with $\mathrm{L}=3$ can outperform theoretical 16-ary PSK signal reaching up to $5.2 \mathrm{~dB}$ at high SNR.

According to the experiment, the case of $\mathrm{L}=2$ greatly reduces the probability of error than the case of $\mathrm{L}=1$ and the calculation time is at an acceptable level. Taking into account the calculation time and BER, $\mathrm{L}=2$ is preferable. If the computing time is sufficient, the $\mathrm{BER}$ in the case of $\mathrm{L}=3$ is further reduced.

\section{Conclusions}

This paper has built a Markov Chain model to evaluate the autocorrelation function and get the M-ary MSK by Fourier transform for M-ary MSK. And numerical signal simulations verify the correctness of the results in those figures. It has been shown that the composition of each level $\mathrm{M}$ spectrum analyzes the characteristics of the spectrum. The fractional out-of-band power of each M-ary MSK that $\mathrm{M}=2,4,8,16$ has been compared, which shows that when $\mathrm{BT}_{b}>0.71$, 4-ary MSK has significantly less out-of-band power compared to another communication system. We adopt OMLCD to receive the signal and draw the BER curves at different SNRs. For inherent memory L, we can conclude that when L changes from 1 to 2, the BER improves about $3 \mathrm{~dB}$. When $\mathrm{L}=3$, the BER improves about $4 \mathrm{~dB}$. With the increase of MSK level, except binary MSK, the BER is $4 \mathrm{~dB}$ higher than the theoretical PSK level.

\section{Appendix 1}

In appendix 1, examples of MSK, 4-ary MSK and 8-ary MSK matrix are listed. Example: MSK: 


$$
P_{M=2}^{1}=A P_{M=2}^{2}=A^{2}
$$

4-ary MSK:

$$
P_{M=4}^{1}=\left(\begin{array}{ll}
A & A \\
B & B
\end{array}\right) P_{M=4}^{2}=\left(\begin{array}{ll}
A^{2}+A B & A^{2}+A B \\
A B+B^{2} & A B+B^{2}
\end{array}\right)
$$

8-ary MSK:

$$
P_{M=8}{ }^{1}=\left(\begin{array}{cccc}
A & A & A & A \\
B & B & B & B \\
A & A & A & A \\
B & B & B & B
\end{array}\right) P_{M=8}{ }^{2}=2 *\left(\begin{array}{llll}
A^{2}+A B & A^{2}+A B & A^{2}+A B & A^{2}+A B \\
A B+B^{2} & A B+B^{2} & A B+B^{2} & A B+B^{2} \\
A^{2}+A B & A^{2}+A B & A^{2}+A B & A^{2}+A B \\
A B+B^{2} & A B+B^{2} & A B+B^{2} & A B+B^{2}
\end{array}\right)
$$

\section{Appendix 2}

In appendix 2 shows the details of deriving the PSD function.

\subsection{Case 1: Where $0 \leq \mathrm{t} \leq \mathrm{T}$ and $\mathrm{m}=0, S_{m=0}^{M}$}

$$
\begin{aligned}
& S^{M}{ }_{m=0}(f)=\sum_{i=1}^{N} \sum_{j=1}^{N} \int_{-\infty}^{\infty} 2 P(\mathrm{i})\left[\mathrm{p}(\mathrm{j} \mid \mathrm{i}, \mathrm{m}) \mathrm{s}_{i j}\left(\mathrm{~T}-\tau^{\prime}\right)\right] \cos 2 \pi f \tau d \tau+\sum_{i=1}^{N} \sum_{j=1}^{N} \int_{-\infty}^{\infty} 2 P(\mathrm{i})\left[\mathrm{p}(\mathrm{j} \mid \mathrm{i}, \mathrm{m}+1) \mathrm{s}_{j i}{ }^{*}\left(\tau^{\prime}\right)\right] \cos 2 \pi f \tau d \tau \\
& =2 \int_{0}^{T_{b}} R 1^{M}{ }_{m=0}(\tau) \cos 2 \pi f \tau d \tau+2 \int_{0}^{T_{b}} R 2^{M}{ }_{m=0}(\tau) \cos 2 \pi f \tau d \tau \\
& =2 \int_{0}^{T_{b}} R 1^{M}{ }_{m=0}(\tau) \cos 2 \pi f \tau d \tau+2 \int_{0}^{T_{b}} R 2_{-} \operatorname{same}^{M}{ }_{m=0}(\tau) \cos 2 \pi f \tau d \tau+2 \int_{0}^{T_{b}} R 2_{-} d i f^{M}{ }_{m=0}(\tau) \cos 2 \pi f \tau d \tau \\
& =S 1^{M}{ }_{m=0}(f)+S 2 \_ \text {same }^{M}{ }_{m=0}(f)+S 2_{-} \operatorname{dif}^{M}{ }_{m=0}(f)
\end{aligned}
$$

$S^{M}{ }_{m=0}(f)$ is divided into $S 2_{-} \operatorname{same}^{M}{ }_{m=0}(f), S 1^{M}{ }_{m=0}(f)$ and $S 2_{-} \operatorname{dif}^{M}{ }_{m=0}(f)$ for the summation calculation.

$$
\begin{aligned}
S 1^{M}{ }_{m=0}(f) & =2 \int_{0}^{T_{b}} \frac{1}{2}\left(1-\frac{\tau}{T_{b}}\right) \cos \frac{(2 n-1) \pi \tau}{2 T_{b}} \cos 2 \pi f \tau d \tau \\
= & \int_{0}^{T_{b}} \cos \frac{n \pi \tau}{2 T_{b}} \cos 2 \pi f \tau d \tau-\int_{0}^{T_{b}} \frac{\tau}{T_{b}} \cos \frac{n \pi \tau}{2 T_{b}} \cos 2 \pi f \tau d \tau \\
= & T\left\{\frac{\sin [\varphi(n)+\omega(f)]}{2[\varphi(n)+\omega(f)]}+\frac{\sin [\varphi(n)-\omega(f)]}{2[\varphi(n)-\omega(f)]}-\frac{\cos [\varphi(n)-\omega(f)]+[\varphi(n)-\omega(f)] \sin [\varphi(n)-\omega(f)]-1}{2[\varphi(n)-\omega(f)]^{2}}\right. \\
- & \left.\frac{\cos [\varphi(n)+\omega(f)]+[\varphi(n)+\omega(f)] \sin [\varphi(n)+\omega(f)]-1}{2[\varphi(n)+\omega(f)]^{2}}\right\} \\
S 2 \_\operatorname{same}^{M}{ }_{m=0}(f) & =2 \int_{0}^{T_{b}} \frac{1}{2} \frac{\tau^{\prime}}{T_{b}} \sin \frac{\pi\left(T_{b}-\tau^{\prime}\right) n}{2 T_{b}} \cos 2 \pi f \tau d \tau+2 \int_{0}^{T_{b}} \frac{\sin \left(\frac{1}{2} \pi n\right) \sin \frac{\pi \tau^{\prime} n}{2 T_{b}}}{\pi n} \cos 2 \pi f \tau d \tau \\
& =4 T \frac{\varphi(n)\left[\cos \omega(f) * S_{2}+8 \omega(f) \sin \omega(f)\right]-\sin \varphi(n) * S_{1}(n)}{S_{2}^{2}(n)} \\
& -4 T \frac{\sin \varphi(n)[\omega(f) \sin \omega(f) \sin \varphi(n)+\varphi(n) \cos \omega(f) \cos \varphi(n)-\varphi(n)]}{\varphi(n) S_{2}(n)}
\end{aligned}
$$




$$
\begin{aligned}
S 2 \_d i f^{M}{ }_{m=0}(f) & =4 \int_{0}^{T_{b}} \frac{n\left[\sin \left(\frac{1}{2} \pi m\right) \sin \frac{\pi \tau^{\prime} n}{2 T_{b}}\right]}{\pi\left[m^{2}-n^{2}\right]} \cos 2 \pi f \tau d \tau-4 \int_{0}^{T_{b}} \frac{m\left[\cos \frac{\pi\left(T_{b}-\tau^{\prime}\right) m}{2 T}\right]}{\pi\left[m^{2}-n^{2}\right]} \cos 2 \pi f \tau d \tau \\
& =8 T \frac{\varphi(n) \sin \varphi(m) *[\omega(f) \sin \omega(f) \sin \varphi(n)+\varphi(n) \cos \omega(f) \cos \varphi(n)-\varphi(n)]}{[\varphi(n)-\varphi(m)][\varphi(n)+\varphi(m)] S_{2}(n)} \\
& +8 T \varphi(m) \frac{\varphi(m) \sin \varphi(m)-\omega(f) \sin \omega(f)}{[\varphi(n)-\varphi(m)][\varphi(n)+\varphi(m)] S_{2}(m)}
\end{aligned}
$$

\subsection{Case 2: Where $\mathrm{T} \leq \mathrm{t} \leq 2 \mathrm{~T}$ and $\mathrm{m}=1, S_{m=1}^{M}$}

$$
\begin{aligned}
S^{M}{ }_{m=1} & =\sum_{i=1}^{N} \sum_{j=1}^{N} \int_{-\infty}^{\infty} 2 P(\mathrm{i})\left[\mathrm{p}(\mathrm{j} \mid \mathrm{i}, \mathrm{m}) \mathrm{s}_{i j}\left(\mathrm{~T}-\tau^{\prime}\right)\right] \cos 2 \pi f \tau d \tau \\
& =2 \int_{T_{b}}^{2 T_{b}} R 1_{-} \operatorname{same}^{M}{ }_{m=1}(\tau) \cos 2 \pi f \tau d \tau+2 \int_{T_{b}}^{2 T_{b}} R 1_{-} \operatorname{dif}^{M}{ }_{m=1}(\tau) \cos 2 \pi f \tau d \tau \\
& =S 1_{-} \text {same }^{M}{ }_{m=1}(f)+S 1 \_d i f^{M}{ }_{m=1}(f)
\end{aligned}
$$

$S^{M}{ }_{m=1}$ is divided into $S 1_{-} \operatorname{same}^{M}{ }_{m=1}(f)$ and $S 1_{-} \operatorname{dif}^{M}{ }_{m=1}(f)$ for the summation calculation.

$S 1_{-} \operatorname{same}^{M}{ }_{m=1}(f)=2 \int_{T_{b}}^{2 T_{b}} \sin \frac{\pi\left(\tau-T_{b}\right) n}{2 T_{b}} \cos 2 \pi f \tau d \tau$

$$
\begin{aligned}
& -\int_{T_{b}}^{2 T_{b}} \frac{\tau}{T_{b}} \sin \frac{\pi\left(\tau-T_{b}\right) n}{2 T_{b}} \cos 2 \pi f \tau d \tau-2 \int_{T_{b}}^{2 T_{b}} \frac{\sin \left(\frac{1}{2} \pi n\right) \sin \frac{\pi\left(2 T_{b}-\tau\right) n}{2 T_{b}}}{\pi n} \cos 2 \pi f \tau d \tau \\
& =-2 T \frac{2[\varphi(n)+\omega(f)] \cos [\varphi(n)-2 \omega(f)]+2[\varphi(n)-\omega(f)] \cos [\varphi(n)+2 \omega(f)]-4 \varphi(n) \cos \omega(f)}{S_{2}(n)} \\
& +2 T\left\{\frac{2[\varphi(n)-\omega(f)] \cos [\varphi(n)-2 \omega(f)]-\sin [\varphi(n)-2 \omega(f)]}{4[\varphi(n)-\omega(f)]^{2}}\right. \\
& \left.+\frac{[\varphi(n)+\omega(f)] \cos [\varphi(n)+2 \omega(f)]-\sin [\varphi(n)+2 \omega(f)]}{4[\varphi(n)+\omega(f)]^{2}}-\frac{2 \varphi(n)\left[S_{2} \cos \omega(f)+8 \omega(f) \sin \omega(f)\right]}{S_{2}^{2}(n)}\right\} \\
& +2 T \sin \varphi(n)\left[\frac{\cos [\varphi(n)-\omega(f)]+\cos [\varphi(n)+\omega(f)]-2 \cos 2 \omega(f)}{S_{2}(n)}-\frac{2 \omega(f) \sin \omega(f) \sin \varphi(n)}{\varphi(n) S_{2}(n)}\right]
\end{aligned}
$$

$$
\begin{aligned}
S 1 \_d i f^{M}{ }_{m=1}(f) & =2 \int_{T_{b}}^{2 T_{b}} \frac{[n-m] \cos \frac{\pi\left[T_{b} m+(2 T-\tau) n\right]}{2 T_{b}}}{\pi\left[m^{2}-n^{2}\right]} \cos 2 \pi f \tau d \tau \\
& -2 \int_{T_{b}}^{2 T_{b}} \frac{2 n \cos \frac{\pi\left(\tau-T_{b}\right) m}{2 T_{b}}}{\pi\left[m^{2}-n^{2}\right]} \cos 2 \pi f \tau d \tau+2 \int_{T_{b}}^{2 T_{b}} \frac{[m+n] \cos \frac{\pi\left(T_{b} m+\left(\tau-2 T_{b}\right) n\right)}{2 T_{b}}}{\pi\left[m^{2}-n^{2}\right]} \cos 2 \pi f \tau d \tau \\
& =\frac{T}{2}\left\{\frac{\sin [\varphi(m)+2 \omega(f)]-\sin [\varphi(n)+\varphi(m)+\omega(f)]}{[\varphi(n)+\varphi(m)][\varphi(n)-\omega(f)]}-\frac{\sin [2 \omega(f)-\varphi(m)]+\sin [\varphi(n)+\varphi(m)-\omega(f)]}{[\varphi(n)+\varphi(m)][\varphi(n)+\omega(f)]}\right\} \\
& -T \varphi(n) \frac{[\varphi(m)-\omega(f)] \sin [\varphi(m)+2 \omega(f)]+[\varphi(m)+\omega(f)] \sin [\varphi(m)-2 \omega(f)]+2 \omega(f) \sin \omega(f)}{\left[\varphi^{2}(m)-\varphi^{2}(n)\right]\left[\varphi^{2}(m)-\omega^{2}(f)\right]} \\
& +T\left\{\frac{\sin [\varphi(m)+2 \omega(f)]-\sin [\varphi(m)-\varphi(n)+\omega(f)]}{2[\varphi(m)-\varphi(n)][\varphi(n)+\omega(f)]}-\frac{\sin [\varphi(m)-\varphi(n)-\omega(f)]-\sin [\varphi(m)-2 \omega(f)]}{2[\varphi(m)-\varphi(n)][\varphi(n)-\omega(f)]}\right\}
\end{aligned}
$$




\section{References}

[1] Chen, Xingbo, Liu, et al., "Energy leakage analysis of the radar and communication integrated waveform,” Iet Signal Processing, 12(3), 375-382, 2018. Article (CrossRef Link)

[2] Dong S, Momson I, Kshattry S, et al., "A 10-Gb/s 180-GHz Phase-Locked-Loop Minimum Shift Keying Receiver,” IEEE Journal of Solid-State Circuits, 56(3), 681-693, 2021. Article (CrossRef Link)

[3] Wang Y, Zhang A, Ma J, "Research on the system performance evaluation of minimum-shift keying in uplink ground-to-satellite with gamma-gamma distribution,” Optical Engineering, 56(7), 076113, 2017. Article (CrossRef Link)

[4] Pelchat M G, "The Autocorrelation Function and Power Spectrum of PCM/FM with Random Binary Modulating Waveforms,” IEEE Transactions on Space Electronics \& Telemetry, 10(1), 3944, 1964. Article (CrossRef Link)

[5] Le N B, "Multi-amplitude minimum shift keying modulation format for optical communications," Optics Communications, 281(17), 4245-4253, 2008. Article (CrossRef Link)

[6] Tao S, Chen J, Yang Y, et al., "Study of a novel amplitude-phase modulation IRZ-MSK in highspeed optical fiber transmission system,” Optical Fiber Technology, 20(3), 254-260, 2014. Article (CrossRef Link)

[7] Gao C, Haimovich A M, "BER analysis of MPSK space-time block codes with differential detection,” IEEE Communications Letters, 7(7), 314-316, 2003. Article (CrossRef Link)

[8] Anderson R R, Salz J, “Spectra of Digital FM,” Bell System Technical Journal, 44(6), 1165-1189, 1965. Article (CrossRef Link)

[9] Bennett W R, Rice S O, "Spectral Density and Autocorrelation Functions Associated with Binary Frequency-Shift Keying,” Bell System Technical Journal, 42(5), 2355-2385, 1963.

Article (CrossRef Link)

[10] Ekanayake N, "M-ary continuous phase frequency shift keying with modulation index 1/M," Iee Proceedings F Communications Radar \& Signal Processing, 131(2), 173-178, 1984.

Article (CrossRef Link)

[11] Rimoldi B E, “A decomposition approach to CPM,” IEEE Transactions on Information Theory, 34(2), 260-270, 1998. Article (CrossRef Link)

[12] Robert CP, Casella G., Monte Carlo statistical method, New York: Springer, 2004.

[13] Douc R, Moulines E, Priouret P, et al., Markov Chains, 2018.

Article (CrossRef Link)

[14] Gupta A, Mukherjee B, Upadhyay S K, "Weibull extension model: A Bayes study using Markov chain Monte Carlo simulation,” Reliability Engineering \& System Safety, 93(10), 1434-1443, 2008. Article (CrossRef Link)

[15] Simon M K, Divsalar D, "On the optimality of classical coherent receivers of differentially encoded M-PSK,” IEEE Communications Letters, 1(3), 67-70, 1997. Article (CrossRef Link)

[16] Park H C, Lee K, Feher K, “Continuous Phase Modulation of F-QPSK-B Signals,” IEEE Transactions on Vehicular Technology, 56(1), 157-172, 2007. Article (CrossRef Link)

[17] Andersson S T, Svensson N A B, "Noncoherent detection of convolutionally encoded continuous phase modulation,” IEEE Journal on Selected Areas in Communications, 7(9), 1402-1414, 1989. Article (CrossRef Link)

[18] Jung P, Baier P W, "On the representation of CPM signals by linear superposition of impulses in the bandpass domain,” IEEE Journal on Selected Areas in Communications, 10(8), 1236-1242, 1992. Article (CrossRef Link)

[19] Hamed A M, Rao R K, "Energy and spectral efficiencies of M-CPFSK in fading and shadowing wireless channels,” Physical Communication, 30, 204-212, 2018. Article (CrossRef Link)

[20] Serfozo Richard, Basics of Applied Stochastic Processes, Springer, 2009. Article (CrossRef Link)

[21] J.G. Proakis, M. Salehi, “Digital Communications,” Digital Communications, 73(11), 3-5, 2015. 


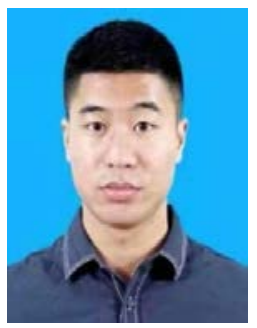

Zheng Niu was born in Hefei, Anhui in 1997. He received a bachelor's degree in electronics and communication engineering from Nanchang University in 2019.

He is currently pursuing a master's degree in Information and Communication Engineering with the School of Wuhan Naval University of Engineering, Hubei

His research interests include Very Long Wave Communication, modulation and demodulation, swarm particle, swarm optimization, genetic algorithms.

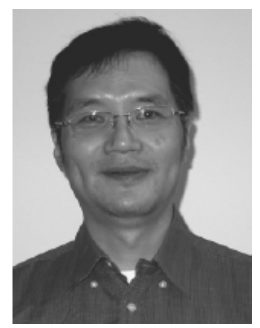

Yuzhong Jiang was born in Jiaxing, China, in 1963. He received the B.S. degree in electrical engineering from the Naval University of Engineering at Wuhan Hubei, China, in 1981, the M.S. degree in electrical engineering from Nanjing University of Aeronautics and Astronautics, in 1990, and the Ph.D. degree in electrical and information engineering from the University of Science and Technology Huazhong, Wuhan, China, in 2008. From 2005 to 2006, he was a senior visiting scholar at Tsinghua University. Since 2007, he has been a Professor with the College of Electrical Engineering of the Naval University of Engineering at Wuhan Hubei, China. His general research interest lies in statistical methods for signal processing with applications in communications.

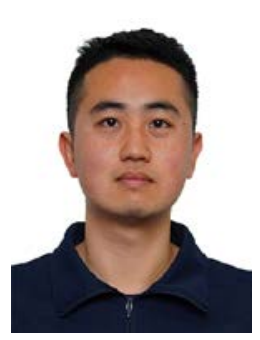

Shuyang Jia was born in 1994, received the master's degree in 2020 from the Naval Engineering University, Wuhan, in information and communication engineering Academy. $\mathrm{He}$ is currently working toward the Ph.D. degree in ship and ocean engineering of Submarine Academy, His research interest is in the area of information theory and coding in underwater acoustic communication.

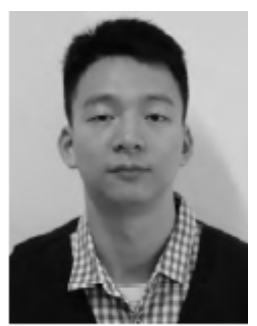

Huang Zhi was born in Fujian, China in 1994. He received a bachelor's degree in information countermeasures from Wuhan Naval University of Engineering, Hubei in 2017, and a master's degree in circuits and systems in 2019. He is currently studying for a doctorate in information and communication engineering. His research interests include radiation source identification, deep learning, and non-Gaussian noise modeling.

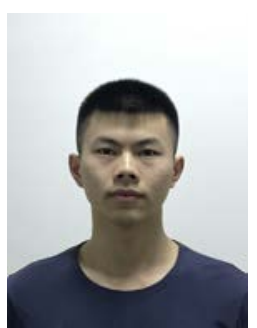

Wenliang Zou, born in Zhangshu, Jiangxi in 1998, received a bachelor's degree in electronics and communication engineering from Nanchang University in 2020. He is currently a graduate student and his main research field is communication and information systems and applications. 


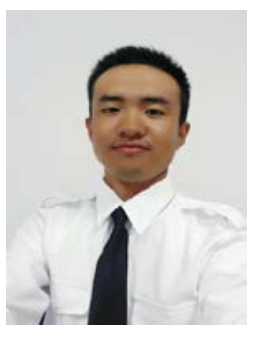

Gang Liu was born in 1994, male, Mianyang, Sichuan, Naval Engineering University, student, master's degree, research direction: signal processing and communication noise reduction.

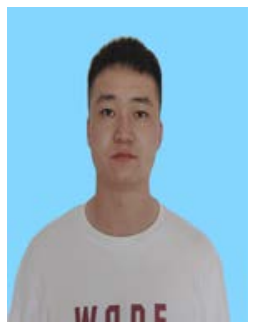

Yu Li was born in 1995, male, Zhangye, Gansu, received a bachelor's degree in electronics and communication engineering from Nanchang University in 2017. Research direction: signal processing. 